intubation: group A (antegrade) and group B (retrograde). The primary outcome variable of the study was the successful completion of the procedure. Failed procedure is defined as the procedure can not be completed by using PBS technique or sedation-related serious adverse events such as severe hypoxaemia $\left(\mathrm{SpO}_{2}<85 \%\right.$ more than $3 \mathrm{~min}$ and can not relief by airway management), severe cardiorespiratory instability, are occurred. The secondary outcome variables were sedation-related complications, mortality rate and haemodynamic parameters.

Results 108 patients underwent SBE procedure during the study period. After matching age, gender, weight, height, ASA physical status, duration of endoscopy and indications of procedures, there were 21 patients in group $A$ and 19 patients in group $B$. There were no significant differences in age, gender, weight, height, ASA physical status, duration and indication of procedures, type of enteroscopy, anaesthetic personnel and haemodynamic parameters between the two groups. All procedures were successful completion of the endoscopies. Mean dose of propofol, fentanyl and midazolam in both groups was comparable. Overall and cardiorespiratoryrelated adverse events were not significantly different between the two groups. All adverse events were transient, mild degree and easier treatable. Serious adverse events were none.

Conclusion PBA for SBE procedure in adult patients by experienced anaesthesiologist is relative safe and effective. The success rate of the endoscopy does not depend on the route of intubation. Serious adverse events were rare in our population.

Competing interests None declared.

\section{PWE-206 PROPOFOL DEEP SEDATION FOR ELDERLY PATIENTS: A COMPARISON BETWEEN EUS WITH OR WITHOUT FINE NEEDLE ASPIRATION PROCEDURE}

doi:10.1136/gutjnl-2012-302514d.206

S Amornyotin,* W Chalayonnawin, S Kongphlay. Department of Anesthesiology, Faculty of Medicine Siriraj Hospital, Mahidol University, Bangkok, Thailand

Introduction The aim of this study was to evaluate and compare the clinical efficacy of propofol deep sedation (PDS) for elderly patients underwent EUS with or without fine needle aspiration (FNA) procedure in a teaching hospital in Thailand.

Methods We undertook a retrospective review of the sedation service records of patients who underwent EUS procedures from December 2006 and September 2009. All patients were classified into two groups according to the type of procedure. In group A, EUS was only done for diagnosis. In group $\mathrm{B}$, EUS with FNA was done. The primary outcome variable of the study was overall complication rate. The secondary outcome variables were sedation and procedurerelated complications during and immediately after the procedure and haemodynamic parameters.

Results PDS was provided for 513 patients. After matching age, gender, weight and ASA physical status, there were 47 patients in group A, and 40 patients in group B. There were no significantly differences in age, gender, weight, ASA physical status, mean sedative agents used, and indications of endoscopy between the two groups. However, duration of procedure in group B was significantly longer than in group A. All patients in both groups were concluded with the successful completion of the procedure. There were no significant differences in overall complication rate, sedation and procedure-related complications as well as haemodynamic parameters among the two groups. All complications were easily treated, with no adverse sequelae.

Conclusion PDS for EUS with or without FNA procedure in elderly patients by trained anaesthetic personnel with appropriate monitoring was relatively safe and effective. Complications in both groups were comparable. Serious complications were rare in our population.
Competing interests None declared.

\section{PWE-207 BILE DUCT LEAKS FROM AN ABERRANT DUCT OF LUSCHKA}

doi:10.1136/gutjnl-2012-302514d.207

S Thayalasekaran, ${ }^{*}$ M Gibson, J Kinchen, J Booth, M Booth, A Mee. Department of Gastroenterology, NHS, Reading, UK

Introduction The Duct of Luschka is an accessory biliary radicle first described by the German anatomist Hubert von Luschka in the 19th century. If this aberrant duct goes unnoticed at the time of cholecystectomy, the patient is likely to develop a biliary leak postoperatively. The majority of post-operative leaks are from the cystic duct remnant and standard management is ERCP and stent insertion across the cystic duct $+/$-sphincterotomy. The aim of our study was to identify the number of leaks from an aberrant "Duct of Luschka" in patients who underwent laparoscopic cholecystectomy over a 14-year period and to evaluate the efficacy of ERCP in their management.

Methods This retrospective study included all patients undergoing cholecystectomy between 1994 and 2010. Those who had a subtotal cholecystectomy were excluded. ERCP reports were reviewed to identify the number of biliary leaks. The medical notes of each patient with an aberrant duct leak were reviewed to evaluate their management.

Results For 5221 laparoscopic cholecystectomies there were 97 biliary leaks (1.9\%), 86 from the cystic duct remnant and 11 from an aberrant duct. In two patients found to have leaks from a Duct of Luschka during surgery, the laparoscopic approach was converted to an open procedure and one patient had a drain inserted at initial laparoscopy. Eight patients had a repeat laparoscopy with a washout and drain insertion. Two patients had CT guided drain insertion. Time to ERCP after presentation varied from 1 to 10 days, with the majority being done between day two and day five. Of the eleven, four patients had a sphincterotomy and stent insertion, five had stent insertion alone and two patients had no therapeutic intervention as the leak was felt to be too small. Eight patients had a repeat ERCP with stent removal and no residual leak on cholangiogram. One person was lost to follow-up. No patients required surgery after ERCP to control the leak. Arrow below: leaking aberrant Duct of Luschka.

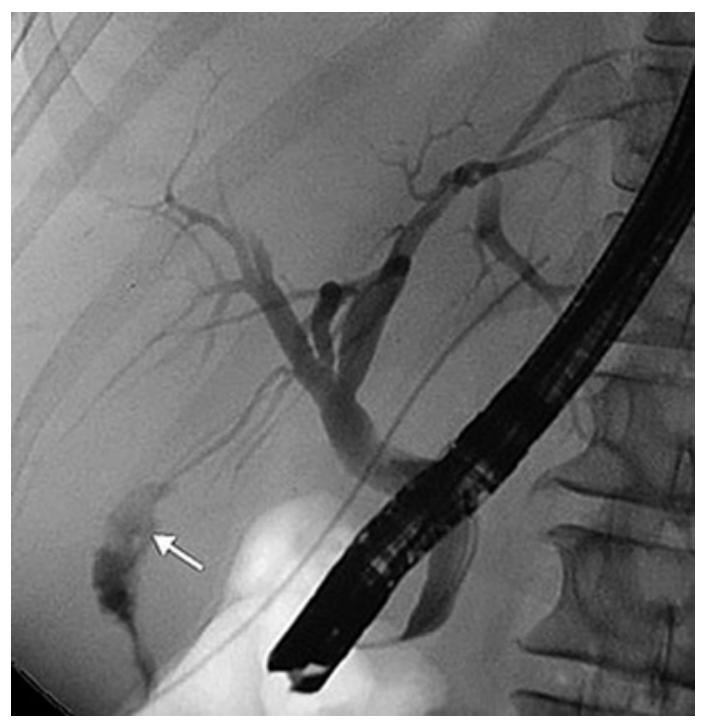

Abstract PWE-207 Figure 1 
Conclusion In this study the likelihood of a post cholecystectomy bile leak arising from an aberrant duct of Luschka was $13 \%$, in keeping with previous smaller series where the rate ranged from $0 \%$ to $21 \%$. The standard managment with stent insertion +/sphincterotomy appears to be effective even though the leak is not covered by the stent, presumably by providing preferential drainage.

Competing interests None declared.

\section{REFERENCES}

1. Tantia 0, Jain M, Khanna S, et al. latrogenic biliary injury. Surg Endosc 2008;22:1077-86.

2. Sharma H, Bird G. Endoscopic management of postcholecystectomy biliary leaks. Frontline Gastroenterol 2011;2:230-3.

3. Bartofi J, Baranya F, Simon E, et al. [Laparoscopic treatment of bile leakage from the Luschka Duct after laparoscopic cholecystectomy]. Orv Hetil 2004;145:1061-4.

\section{PWE-208 HIGH PREVALENCE OF HYPERPLASTIC POLYPOSIS SYNDROME IN THE NHS BOWEL CANCER SCREENING PROGRAMME}

doi:10.1136/gutjnl-2012-302514d.208

${ }^{1} \mathrm{~S}$ Biswas, ${ }^{*} \mathrm{~A} J$ Ellis, ${ }^{2} \mathrm{R}$ Guy, ${ }^{3} \mathrm{R}$ Chetty, ${ }^{4} \mathrm{~K}$ Madronal, ${ }^{4} \mathrm{H}$ Savage, ${ }^{1} \mathrm{~J}$ E East. ${ }^{1}$ Translational Gastroenterology Unit, Oxford University Hospitals NHS Trust, Oxford, UK; ${ }^{2}$ Colorectal Surgery, Oxford University Hospitals NHS Trust, Oxford, UK; ${ }^{3}$ Translational Pathology, Oxford University Hospitals NHS Trust, Oxford, UK; ${ }^{4}$ Bowel Cancer Screening, Oxford University Hospitals NHS Trust, Oxford, UK

Introduction Hyperplastic Polyposis Syndrome (HPS) is a rare syndrome (estimated 1:3000, 0.033\% general population ${ }^{1}$ ) in which multiple hyperplastic polyps can predispose to an increased risk of colorectal cancer of up to $7 \%$ at 5 years, ${ }^{2}$ and a risk for first degree relatives of HPS patients of fivefold compared to the general population. ${ }^{3}$ Proximal serrated polyps are commonly associated with advanced neoplasia. ${ }^{4}$ Currently the Bowel Cancer Screening Programme (BCSP) does not offer surveillance for serrated polyps. We aimed to assess how common HPS is in our BCSP population. Methods We reviewed endoscopic and pathology records for all patients presenting for Bowel Cancer Screening in Oxfordshire between April 2010 (programme start) and January 2012. Three endoscopists performed the procedures. Patients were defined as HPS if they met either of the two main WHO criteria for HPS: either $\geq 20$ hyperplastic polyps throughout the colon, or five hyperplastic polyps in the proximal colon with $2 \geq 10 \mathrm{~mm}$. Patients who were 1st degree relatives of HPS patients were not considered. Results In total 755 patients attended for screening colonoscopy. Five patients met WHO criteria for HPS, of whom three had a synchronous advanced adenoma (see Abstract PWE-208 table 1). The prevalence of HPS in our BCSP population was $0.66 \%$ (95\% CI $0.24 \%$ to $1.52 \%$ ), a 20 -fold increase compared to the estimated rate in the general population.

Conclusion HPS appears to be relatively common in BCSP patients and is often associated with advanced neoplasia. Detection of a large serrated polyp or multiple hyperplastic polyps should alert BCSP colonoscopists to the possibility of HPS where they may wish to augment detection with dye-spray or advanced imaging techniques. BCSP surveillance for large proximal serrated polyps may need to be reviewed to ensure such patients are not overlooked.

Competing interests None declared.

\section{REFERENCES}

Lockett MJ, Atkin WS. Gut 2001; 48(Suppl I):A4. 18.

Boparai KS, et al. Gut 2010;59:1094-100.

Boparai KS, et al. Gut 2010;59:1222-5.

Hiraoka S, et al. Gastroenterology 2010;139:1503-10.

\section{PWE-209 ENDOSCOPY INCREASES THE RISK OF VENOUS}

doi:10.1136/gutjnl-2012-302514d.209

${ }^{1} \mathrm{~S}$ V Venkatachalapathy, ${ }^{*}{ }^{1} \mathrm{P}$ Kiwanuka-musoke, ${ }^{2} \mathrm{G}$ Evans, ${ }^{3} \mathrm{P}$ Bassett, ${ }^{1} \mathrm{~A}$ F Muller ${ }^{1}$ Department of Gastroenterology, East Kent Hospitals University NHS Foundation Trust, Canterbury, UK; ${ }^{2}$ Department of Haematology, East Kent Hospitals University NHS Foundation Trust, Canterbury, UK; ${ }^{3}$ Department of Statistics, Statsconsultancy, Amersham, UK

Introduction Venous thromboembolism (VTE) is a major cause of mortality contributing up to 25000 deaths per year. There are no published studies that have linked the risk of VTE to endoscopic procedures. The current study was designed to assess whether endoscopic procedures increase the risk of venous thrombosis.

Methods A retrospective case-control study of cases of patients (pts) with VTE from the Trust's Haemophilia department database over a 3-year period from 1 January 2009 to 31 December 2011. Each case was age and sex matched to one control patient, who attended an outpatient appointment during the same period. The notes and endoscopy reports of cases and controls were reviewed to identify those patients, who had undergone endoscopy in the preceding 3 months of the VTE diagnosis. All adult patients were included in the study. Pts undergoing OGD, flexible sigmoidoscopy, colonoscopy and ERCP were included. Endoscopic US and bronchoscopy pts were excluded. Study sample size was calculated from a literature review of an approximate $2 \%$ occurrence of endoscopy in the population and our internal pilot study suggesting a $5 \%$ incidence of endoscopy in patients with VTE. With a $5 \%$ significance level and $80 \%$ power we calculated that 425 subjects per group were required to confirm a difference in endoscopy between pts with VTE and controls. The difference in occurrence of endoscopy between cases and controls was examined using the Mc Nemar test. The risk of VTE occurring following endoscopy was quantified using ORs.

Results $45 / 445(10.1 \%)$ patients had endoscopy in the VTE group compared to controls $(14 / 445,3.2 \%$; $<<0.001)$ of which, 21 had OGD, 17 colonoscopy, one had ERCP and six Sigmoidoscopy. The number of pts in each endoscopy procedure category was insufficient to confirm whether the risk of VTE was dependent on the type of procedure performed. The OR for developing a VTE after an endoscopic procedure was 3.58 (95\% CI 1.86 to 7.46).

Abstract PWE-208 Table 1 Characteristics of patients diagnosed with HPS

\begin{tabular}{|c|c|c|c|c|c|c|c|c|}
\hline & $\begin{array}{l}\text { Age at index } \\
\text { colonoscopy }\end{array}$ & Gender & $\begin{array}{l}\text { No } \\
\text { adenomas }\end{array}$ & $\begin{array}{l}\text { Largest adenoma } \\
\text { and location }\end{array}$ & $\begin{array}{l}\text { No hyperplastic } \\
\text { polyps in } \\
\text { whole colon }\end{array}$ & $\begin{array}{l}\text { No hyperplastic } \\
\text { polyps } \geq 10 \mathrm{~mm} \\
\text { in proximal colon* }\end{array}$ & $\begin{array}{l}\text { No hyperplastic polyps } \\
\text { in proximal colon* }\end{array}$ & $\begin{array}{l}\text { No SSPs in } \\
\text { whole colon }\end{array}$ \\
\hline Patient 1 & 69 & $M$ & 7 & $13 \mathrm{~mm}$ sigmoid & 21 & 2 & 12 & 1 \\
\hline Patient 2 & 64 & $\mathrm{~F}$ & 1 & $15 \mathrm{~mm}$ sigmoid & 24 & 5 & 24 & 4 \\
\hline Patient 3 & 62 & M & 8 & $32 \mathrm{~mm}$ sigmoid & 37 & 0 & 5 & 8 \\
\hline Patient 4 & 64 & M & 2 & $8 \mathrm{~mm}$ descending & 43 & 3 & 13 & 5 \\
\hline Patient 5 & 68 & $\mathrm{~F}$ & 0 & - & 6 & 4 & 6 & 6 \\
\hline
\end{tabular}

*Proximal colon is defined as proximal to the recto-sigmoid.

SSP, sessile serrated polyp. 